\title{
Characteristics of Diffusional Kurtosis in Chronic Ischemia of Adult Moyamoya Disease: Comparing Diffusional Kurtosis and Diffusion Tensor Imaging
}

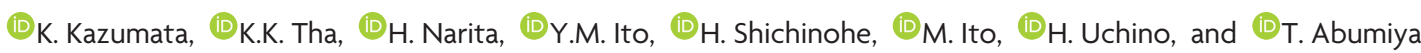

\begin{abstract}
BACKGROUND AND PURPOSE: Detecting microstructural changes due to chronic ischemia potentially enables early identification of patients at risk of cognitive impairment. In this study, diffusional kurtosis imaging and diffusion tensor imaging were used to investigate whether the former provides additional information regarding microstructural changes in the gray and white matter of adult patients with Moyamoya disease.
\end{abstract}

MATERIALS AND METHODS: MR imaging (diffusional kurtosis imaging and DTI) was performed in 23 adult patients with Moyamoya disease and 23 age-matched controls. Three parameters were extracted from diffusional kurtosis imaging (mean kurtosis, axial kurtosis, and radial kurtosis), and 4, from DTI (fractional anisotropy, radial diffusivity, mean diffusivity, and axial diffusivity). Voxelwise analysis for these parameters was performed in the normal-appearing brain parenchyma. The association of these parameters with neuropsychological performance was also evaluated.

RESULTS: Voxelwise analysis revealed the greatest differences in fractional anisotropy, followed, in order, by radial diffusivity, mean diffusivity, and mean kurtosis. In patients, diffusional kurtosis imaging parameters were decreased in the dorsal deep white matter such as the corona radiata and superior longitudinal fasciculus $(P<.01)$, including areas without DTI abnormality. Superior longitudinal fasciculus fiber-crossing areas showed weak correlations between diffusional kurtosis imaging and DTI parameters compared with tissues with a single-fiber direction (eg, the corpus callosum). Diffusional kurtosis imaging parameters were associated with general intelligence and frontal lobe performance.

CONCLUSIONS: Although DTI revealed extensive white matter changes, diffusional kurtosis imaging additionally demonstrated microstructural changes in ischemia-prone deep white matter with abundant fiber crossings. Thus, diffusional kurtosis imaging may be a useful adjunct for detecting subtle chronic ischemic injuries.

ABBREVIATIONS: $A D=$ axial diffusivity; $A K=$ axial kurtosis; $C P T$ = continuous performance task; $D K I=$ diffusional kurtosis imaging; $F A=$ fractional anisotropy; $\mathrm{IQ}=$ intelligence quotient; $\mathrm{MK}=$ mean kurtosis; $\mathrm{MD}=$ mean diffusivity; $\mathrm{MMD}=$ Moyamoya disease; $\mathrm{RD}=$ radial diffusivity; $\mathrm{RK}=$ radial kurtosis; $\mathrm{RST}=$ Reading Span Test; SLF = superior longitudinal fasciculus; TMT $=$ Trail-Making Test

M oyamoya disease (MMD) is characterized by compensatory development of enlarged and weak basal perforating arteries (Moyamoya vessels) due to bilateral occlusive changes in the internal carotid system. ${ }^{1}$ In addition to cerebral ischemia and intracranial hemorrhage, patients with MMD demonstrate neurocognitive issues, such as executive dysfunction, attention deficits,

Received August 26, 2015; accepted after revision January 7, 2016.

From the Departments of Neurosurgery (K.K., H.S., M.I., H.U., T.A.), Radiobiology and Medical Engineering (K.K.T.), Psychiatry (H.N.), and Biostatistics (Y.M.I.), Hokkaido University Graduate School of Medicine, Sapporo, Japan

The authors have no personal or institutional financial interest in the drugs and imaging modalities described herein.

This study was supported by a grant from the Research Committee on Moyamoya Disease, sponsored by the Ministry of Health, Labor, and Welfare of Japan and the Creation of Innovation Centers for Advanced Interdisciplinary Research Areas Programs, Ministry of Education, Culture, Sports, Science, and Technology, Japan. and working-memory disturbances. ${ }^{2,3}$ Brain atrophy may explain cognitive impairment in the absence of infarction, but detection of these changes has been hampered by the limited sensitivity of conventional neuroimaging methods. Diffusion tensor imaging is useful for determining white matter integrity and providing parameters sensitive to changes in axons, myelin, and organelle structures. ${ }^{4,5}$ Indeed, DTI analysis has revealed a widespread decline in white matter integrity in the normal-appearing brain with MMD. ${ }^{3}$ Thus, DTI can detect

\footnotetext{
Please address correspondence to Ken Kazumata, MD, Department of Neurosurgery, Hokkaido University Graduate School of Medicine, North 15 West 7, Kita, Sapporo 060-8638, Japan; e-mail: kazumata@med.hokudai.ac.jp

-- Indicates open access to non-subscribers at www.ajnr.org

三 Indicates article with supplemental on-line appendix and table.

http://dx.doi.org/10.3174/ajnr.A4728
} 
Table 1: Characteristics of the study participants

\begin{tabular}{lccc}
\hline & Control & Moyamoya Disease & $\boldsymbol{P}$ Value \\
\hline No. of subjects & 23 & 23 & - \\
Age (mean) (range) (yr) & $39.0+8.1(25-56)$ & $40.9+9.5(21-58)$ & .48 \\
Sex (F/M) (No. of subjects) & $13: 10$ & $17: 6$ & .35 \\
Risk factor (DM, HT, HL) (No. of subjects) & 0 & 5 & .049 \\
Symptoms (No. of subjects) & - & 13 & \\
$\quad$ Asymptomatic & - & 10 & \\
TIA & - & & \\
\hline
\end{tabular}

Note:-DM indicates diabetes mellitus; HT, hypertension; HL, hyperlipidemia.

early-stage ischemic injury, which potentially predicts future cognitive outcomes. However, DTI is constrained by technical insufficiencies: It is based on the assumption that water molecules diffuse freely and that diffusion can be characterized by a Gaussian distribution. ${ }^{5}$

In addition, the tensor model is based on the observation that in many tissues, water diffusion is anisotropic (ie, the diffusion is more liberal in some directions and more restricted in others). This anisotropic diffusion can be geometrically depicted as an ellipsoid, described by eigenvectors and eigenvalues. This model performs well in regions where fibers are aligned along a single axis. However, it fails in regions with several fiber populations aligned along intersecting axes because it cannot simultaneously map several diffusion maxima. ${ }^{6}$ Furthermore, because hypoxic-ischemic injury induces neurodegeneration and regression of dendrite arborization in gray matter, the diffusion properties of gray matter may also reveal the early stages of ischemic injury. ${ }^{7,8}$ Nevertheless, analyzing isotropic or near-isotropic tissue such as gray matter by DTI may not be valid because its major parameter, fractional anisotropy (FA), reflects structure only if it is spatially oriented. ${ }^{6}$ A more recent method called diffusional kurtosis imaging (DKI) quantifies the deviation of water molecule diffusion from the Gaussian distribution without assuming any specific diffusion model. ${ }^{6,9}$ Its parameters are thought to represent the complexity of tissue microstructure. ${ }^{6}$ Previous studies have suggested that DKI is sufficiently sensitive to detect age-related alterations in white matter microstructure. ${ }^{10,11}$ Furthermore, measurements of diffusion anisotropy by DKI can reveal sexrelated and pathologic changes in gray matter. ${ }^{12,13}$ Thus, using DKI to evaluate the diffusion properties of gray matter and white matter in patients with MMD may be useful for detecting subtle microstructural changes due to ischemia.

Diffusional kurtosis has been investigated to explore tissue reversibility in acute cerebral infarction. ${ }^{14-16}$ However, there is a paucity of information regarding the microstructural properties measured by DKI in chronic ischemia in living humans. To expand on our prior DTI study, we investigated whether adults with MMD and no overt cerebral infarctions have altered diffusional kurtosis in the entire cerebrum. An exploratory voxel-based whole-brain analysis was performed to map regional DKI parameters and to compare DKI and DTI parameters. We also explored correlations of diffusion parameters with measures of neurocognitive impairment in an ROI analysis.
MATERIALS AND METHODS

\section{Participants}

This prospective study was approved by the Research Ethics Committee of Hokkaido University Hospital, and written informed consent was obtained from all participants. Participants in the present study are the same as those of our previous study analyzing the relationship between DTI parameters and neuropsychological test scores. ${ }^{3}$ The selection period was 25 months (April 2012 through April 2014). Twentythree patients ( 6 men and 17 women; $21-58$ years of age; mean age, $40.9 \pm 9.5$ years) were enrolled. The control group also consisted of 23 subjects ( 10 men and 13 women; $25-56$ years of age; mean age, $39.0 \pm 8.1$ years). A brief summary of patient characteristics is provided in Table 1 .

\section{Neuropsychological Assessment}

Neuropsychological examinations consisted of the Wechsler Adult Intelligent Scale-III, Wisconsin Card Sorting Test, TrailMaking Test (TMT; parts A and B), continuous performance task (CPT), Stroop test, and Reading Span Test (RST). The details of neuropsychological examinations and the results are provided in the On-line Appendix. ${ }^{3}$

\section{MR Image Acquisition}

MR imaging was performed with a 3T scanner (Achieva TX; Philips Healthcare, Best, the Netherlands). 3D magnetizationprepared rapid acquistion of gradient echo T1-weighted imaging and axial single-shot spin-echo echo-planar DKI were acquired to evaluate subtle gray and white matter alterations, respectively. The scan parameters for DKI were as follows: TR $=5051 \mathrm{~ms}$, $\mathrm{TE}=85 \mathrm{~ms}$, flip angle $=90^{\circ}, \mathrm{FOV}=224 \times 224 \mathrm{~mm}^{2}$, matrix size $=128 \times 128$, b-values $=0,1000$, and $2000 \mathrm{~s} / \mathrm{mm}^{2}$, number of diffusion gradient directions $=32$, section thickness $=3 \mathrm{~mm}$, intersection gap $=0 \mathrm{~mm}$, number of sections $=43$, and NEX $=1$. The 3D-MPRAGE imaging was performed with $\mathrm{TR}=6.8 \mathrm{~ms}$, $\mathrm{TE}=3.1 \mathrm{~ms}$, flip angle $=8^{\circ}$, and TI $=1100 \mathrm{~ms}$.

\section{Image Processing}

Registration between the echo-planar images with no diffusion weighting $\left(b=0 \mathrm{~s} / \mathrm{mm}^{2}\right)$ and the corresponding DKI data and correction for eddy current distortion were performed at the MR imaging operator console. The DKI data were processed by using Matlab R2012b (MathWorks, Natick, Massachusetts) and Diffusional Kurtosis Estimator (Version 2.5.1; http://nitrc.org/projects/ dke). ${ }^{17}$ Seven DKI and DTI parameters were extracted from the Diffusional Kurtosis Estimator: mean kurtosis (MK), radial kurtosis (RK), axial kurtosis (AK), FA, mean diffusivity (MD), radial diffusivity $(\mathrm{RD})$, and axial diffusivity $(\mathrm{AD})$. The DTI parameters (FA, MD, RD, and $\mathrm{AD}$ ) were calculated from a portion of the DKI data by using a monoexponential model that assumes a Gaussian probability diffusion function by using data from b-values of 0 and $1000 \mathrm{~s} / \mathrm{mm}^{2}{ }^{17}$

Following calculation of DKI and DTI parameters, the $b=0$ echo-planar images were warped to the standardized T2 template 
of SPM8 (http://www.fil.ion.ucl.ac.uk/spm/software/spm12). This transformation matrix was applied to the DKI/DTI parameter map of each patient. The warped DKI/DTI maps were averaged and smoothed with a 6-mm full width at half maximum Gaussian kernel to form customized DKI/DTI templates. Native DKI/DTI maps of all patients and control subjects were then warped to the customized, respective DKI/DTI templates. Individual maps were then smoothed with a $6-\mathrm{mm}$ full width at halfmaximum Gaussian kernel. The warped and smoothed DKI/DTI maps were used for group comparisons between the controls and patients with MMD. To investigate the pathology underlying DKI parameters and the influence of fiber crossings on DTI parameters, we investigated the correlations of DKI and DTI parameters in white matter tracts consisting of either a single fiber direction or crossing fibers (ie, multiple directions). ${ }^{18}$ The ROIs were placed on the genu of the corpus callosum (ie, a structure with a single fiber direction) and deep white matter tracts corresponding to the bilateral superior longitudinal fasciculus (SLF) (multiple fiber directions) by using the JHU white matter atlas available in FSL (http://fsl.fmrib.ox.ac.uk/fsl/fslwiki/). ${ }^{19}$

\section{Data Analysis}

Whole-brain diffusion parameters were compared voxel by voxel between the controls and patients with MMD by using SPM8, which implemented the general linear model. We used the 2-sample $t$ test model, and age was considered a covariate. An explicit mask generated by averaging the normalized CSF space of all participants was applied. To explore group differences across both gray matter and white matter, we set statistical significance at $P<$ .01 without correction for family-wise error and clusters of 50 voxels or more. The number of voxels demonstrating a significant difference between the controls and patients with MMD was extracted. The Pearson product-moment correlation coefficient was calculated to investigate the correlation between the DKI and DTI parameters by using DKI/DTI values extracted from the ROI analysis.

We also investigated whether the significant changes in DKI/ DTI parameters were associated with neuropsychological examination scores. A threshold $T$ value of 2.42 , corresponding to $P<$ .01 without correction for family-wise error, was applied to the $T$ contrast map of DKI/DTI parameters obtained by comparing controls and patients, and binary mask images containing voxels above the threshold value were generated. The DKI/DTI parameter values included in the masks were extracted from DKI/DTI of the patients with MMD. The Pearson product-moment correlation coefficients were used for analyses involving the Wechsler Adult Intelligent Scale-III, TMT (parts A and B and the difference in score between TMT-A and TMT-B [B-A]). Patients were further subgrouped into 2, according to their performance scores, error numbers, and reaction time on the Wisconsin Card Sorting Test, Stroop test, CPT, and RST; and the DKI/DTI parameters were compared between these 2 subgroups by using $t$ tests (Online Appendix). For all correlations and comparisons, a $P$ value $<$ .05 was considered statistically significant to explore the possible relationship between neuropsychological scores and diffusion parameters.

\section{RESULTS}

\section{Spatial Distribution of DTI/DKI Differences on Voxel- Based Analysis}

Areas with decreased MK included the right frontal white matter, bilateral thalami, portions of the occipital white matter, corona radiata, corpus callosum, and portions of frontal and parietal white matter corresponding to the posterior segment of the superior longitudinal fasciculus (Fig 1). The decrease in FA was most extensive within the white matter (161,625 voxels), with its decrease amounting to 12,833 voxels (7.9\%) (Fig 2). Significant MK, $\mathrm{RK}$, and AK decreases were observed in 6180 voxels (3.8\%), 3828 voxels $(2.4 \%)$, and 3043 voxels (1.9\%), respectively. In contrast, 9028 voxels $(5.6 \%)$ showed a significant increase in MD; 10,062 (6.2\%), in $\mathrm{RD}$; and $548(0.3 \%)$, in $\mathrm{AD}$ compared with controls. Figure 1 shows DKI/DTI overlap maps, with MK/RK/AK/FA decreases and $\mathrm{MD} / \mathrm{RD}$ increases. Areas with FA decrease were more extensive than those with MK decrease; however, the posterior segment of the SLF showed a decrease in only MK/RK. The increase in $\mathrm{MD} / \mathrm{RD}$ was remarkable in the corona radiata; however, the posterior segment of the SLF showed a decrease in MK/RK without an increase in MD/RD. Decreased AK was observed without changes in $\mathrm{AD}$ for the bilateral thalami, corona radiata, and portions of the temporo-occipital white matter. There was no cortical gray matter with altered DKI/DTI parameters in patients.

\section{Correlation of DTI and DKI Parameters}

Correlations between DKI and DTI parameters were examined in both the genu of the corpus callosum and the bilateral SLF (Table 2). In the corpus callosum of both controls and patients with MMD, MK correlated positively with FA $(r>0.78, P<.01)$, while inverse correlations with $\mathrm{MK}$ were found for MD/RD/AD $(r<-0.82, P<.001)$. In the corpus callosum, RK correlated inversely with $\mathrm{RD}(r<-0.84, P<.001)$, and AK inversely correlated with $\mathrm{AD}$ of the corpus callosum $(r<-0.64, P<.001)$. A significant correlation between MK and FA was observed in the left SLF of controls, but this was to a lesser degree compared with that in the corpus callosum. No correlation was found between MK and FA in the right SLF of controls. In both controls and patients, correlations between $\mathrm{MK}$ and $\mathrm{MD} / \mathrm{RD} / \mathrm{AD}$ were found in the right and left SLFs, albeit to a lesser degree than corpus callosum correlations. The results of correlation between DKI and DTI parameter values are summarized in Table 2.

\section{Association of DKI/DTI Parameters with Neuropsychological Performance Tests}

The DKI parameter (AK) showed significant correlations with motor intelligence quotient (IQ), full-scale IQ, perceptual organization, and processing speed evaluated on the Wechsler Adult Intelligent Scale-III (Fig $3 A, r>0.42, P<.05$ ). Both DKI (MK) and DTI (FA, MD, and RD) parameters showed significant correlations with the TMT part B (Fig $3 B, r>0.44, P<.05$ ). The Stroop test performance showed moderate positive correlations with $\mathrm{MK}, \mathrm{RK}$, and FA and negative correlations with $\mathrm{MD}, \mathrm{RD}$, and $\mathrm{AD}(P<.05)$. RST was associated with MK and AK $(P<.05)$. The correlations between diffusion parameters and neuropsychological scores (Wisconsin Card Sorting Test, Stroop Test, CPT, and RST) are shown in Table 3. 


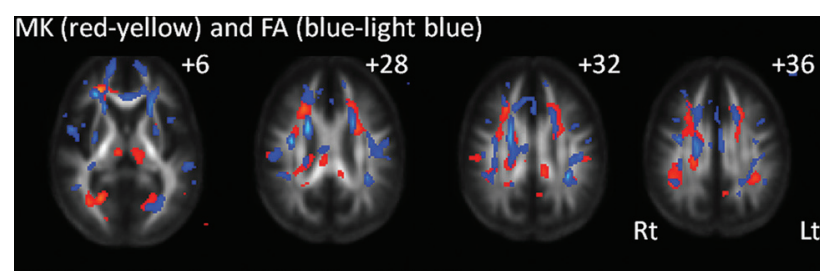

MK (red-yellow) and MD (blue-light blue)

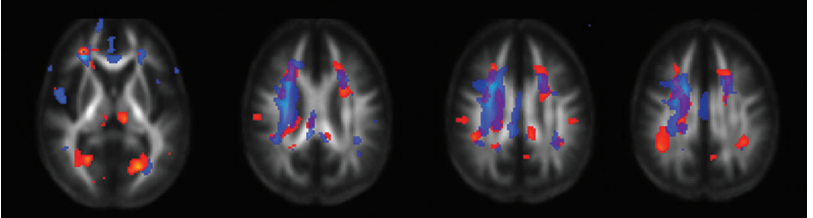

RK (red-yellow) and RD (blue-light blue)

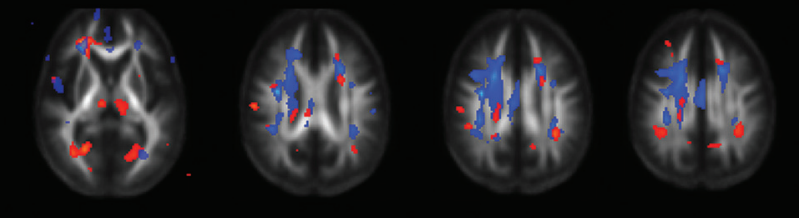

AK (red-yellow) and AD (blue-light blue)

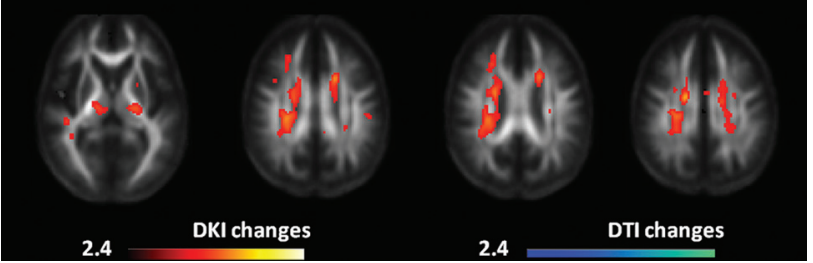

FIG 1. Changes in diffusional kurtosis imaging and diffusion tensor imaging parameters in Moyamoya disease shown in maps of 3 diffusional kurtosis parameters (mean kurtosis, radial kurtosis, and axial kurtosis) and 4 diffusion tensor parameters (fractional anisotropy, mean diffusivity, radial diffusivity, and axial diffusivity). Areas with significant changes in a combination of DKI/DTI parameters are as follows: decreased MK (red-yellow)/decreased FA (blue-light blue), decreased MK (red-yellow)/increased MD (blue-light blue), decreased RK (red-yellow)/increased RD (blue-light blue), and decreased AK (red-yellow)/increased and decreased AD (blue-light blue). Values from statistical parametric mapping analysis are projected onto axial sections of the average brain space of FA ( $z=12,28,32,36 \mathrm{~mm})$. MK decrease is observed in the thalamus, a portion of the genu and body of the corpus callosum, corona radiata, frontoparietal subcortical white matter, and superior longitudinal fasciculus. RK decrease is observed in part of the frontoparietal subcortical white matter, thalamus, corona radiata, and occipital white matter. AK decrease is observed in the thalamus, temporo-occipital white matter, part of the SLF, and corona radiata. The radiologic convention is adopted, with the left side of the brain on the right side of axial panels. The color scale represents $T$ values, with colored regions exceeding the significance threshold of $P<.01(T=2.42)$ with a minimum cluster size of 50 voxels. Rt indicates right; Lt, left.

Table 2: Correlation between DKI and DTI parameters ${ }^{\mathrm{a}}$

\begin{tabular}{|c|c|c|c|c|c|c|c|c|c|c|c|c|}
\hline & \multicolumn{4}{|c|}{ Corpus Callosum } & \multicolumn{4}{|c|}{ Rt. SLF } & \multicolumn{4}{|c|}{ Lt. SLF } \\
\hline & \multicolumn{2}{|c|}{ CNT } & \multicolumn{2}{|c|}{ MMD } & \multicolumn{2}{|c|}{ CNT } & \multicolumn{2}{|c|}{ MMD } & \multicolumn{2}{|c|}{ CNT } & \multicolumn{2}{|c|}{ MMD } \\
\hline & $r$ & $P$ & $r$ & $P$ & $r$ & $P$ & $r$ & $P$ & $r$ & $P$ & $r$ & $P$ \\
\hline MK vs FA & 0.78 & .000 & 0.80 & .000 & 0.08 & .715 & 0.60 & .002 & 0.42 & .047 & 0.47 & .022 \\
\hline MK vs MD & -0.90 & .000 & -0.86 & .000 & -0.60 & .002 & -0.68 & .000 & -0.41 & .049 & -0.49 & .017 \\
\hline MK vs RD & -0.90 & .000 & -0.87 & .000 & -0.54 & .008 & -0.73 & .000 & -0.49 & .018 & -0.55 & .006 \\
\hline MK vs AD & -0.87 & .000 & -0.82 & .000 & -0.50 & .014 & -0.46 & .028 & -0.19 & .386 & -0.35 & .101 \\
\hline RK and RD & -0.88 & .000 & -0.84 & .000 & -0.72 & .000 & -0.56 & .005 & -0.47 & .025 & -0.38 & .072 \\
\hline$A K$ and $A D$ & -0.75 & .000 & -0.64 & .000 & -0.44 & .036 & -0.61 & .001 & -0.47 & .025 & -0.68 & .000 \\
\hline
\end{tabular}

Note:-Rt. indicates right; Lt., left; CNT, controls; $r$; Pearson product-moment correlation coefficient.

${ }^{a}$ All $P$ values, except .715, .386, 101, and ,072, indicate significant correlation between DKI and DTI parameters. 


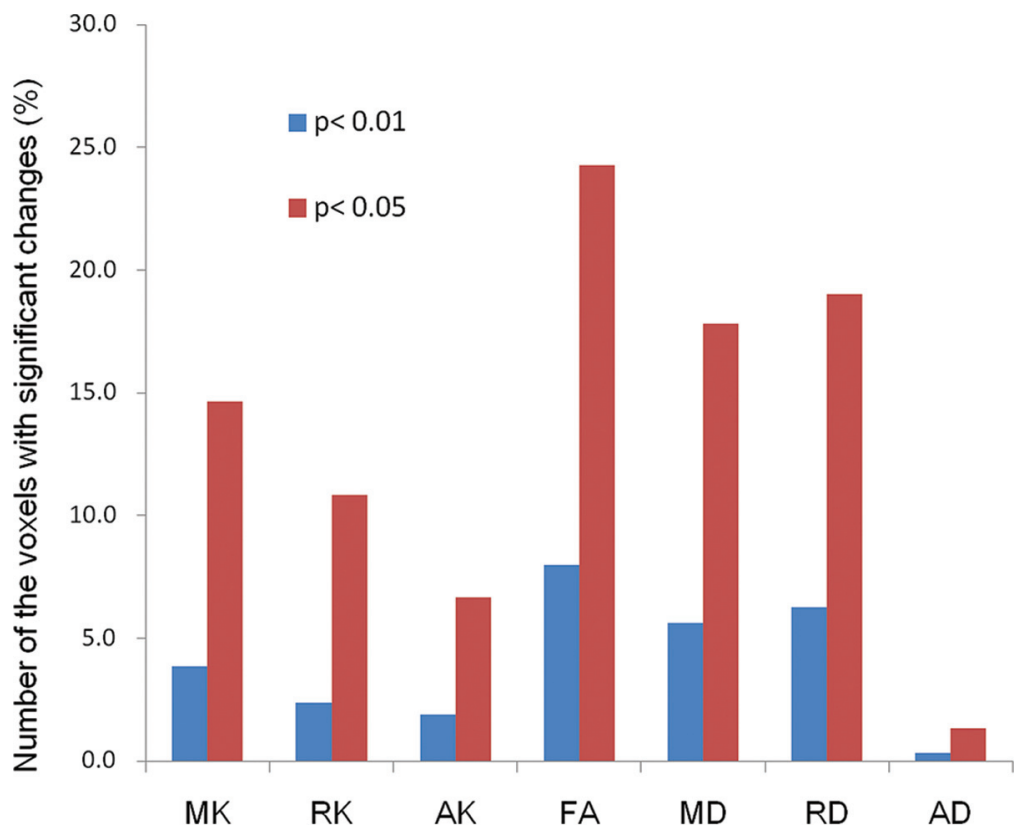

FIG 2. The bar graph indicates the number of the voxels with significant changes relative to the total number of white matter voxels in statistical parametric mapping comparing controls and patients with Moyamoya disease. Bar heights indicate decreases in MK/RK/AK/FA and increases in MD/RD/AD for 2 levels of threshold applied in group comparisons (blue, $P<.01$; red, $P<.05$, respectively).

corona radiata in the voxel-based analysis. Thus, alterations in $\mathrm{AD}$ would be marginal at best in patients, while tissue complexity parallel to the principal diffusion direction could be decreased in the corona radiata of patients. Weak correlations between FA and MK in the SLF of controls could be attributed to the crossing/ kissing of white matter fibers. The SLF contains abundant crossing fibers projecting from the corona radiata and corpus callosum. ${ }^{18}$ Several fiber populations aligned along intersecting axes in 1 voxel would diminish anisotropy. ${ }^{26}$

The DKI parameters in patients showed moderate positive correlations with impaired general intelligence and frontal lobe dysfunction. These results are consistent with previous reports showing a relationship between cognitive performance and white matter fiber tracts integrating parietofrontal cortical areas. ${ }^{27-29}$ In MMD, performance IQ is preferentially affected compared with verbal IQ. ${ }^{1}$ In the present study, AK was significantly correlated with neuropsychological performance on tests evaluating executive function and working memory (motor IQ, perceptual organization, and processing speed), while no correlation was found with verbal IQ. In an experimental animal model of chronic white matter ischemia, damage to myelin preceded axonal damage, suggesting that the change in myelin is the primary pathologic event. ${ }^{30}$ Our observation of a stronger correlation between AK, rather than $\mathrm{AD}$, and general intellectual ability measured by the Wechsler Adult Intelligent Scale-III may imply that a reduction in axonal density and/or axonal degeneration, a more advanced stage of chronic ischemic injury, is better described by AK than AD. Previous investigations by using DTI and probabilistic tractography have shown correlations between neuropsychological examinations and the FA of subcortical white matter. Consistent with previous studies, we observed correlations between DTI parameters and the scores of neuropsychological tests evaluating frontal lobe function in the present study. A significant correlation of FA/ MD/RD with scores on the Trail-Making Test suggests that demyelination alone could affect part of the frontal lobe functions.

Transient ischemic attack followed by hyperventilation is a characteristic symptom of childhood MMD, and syncope attacks are occasionally observed in both children and adults with MMD. ${ }^{1}$ The spatial characteristics of nascent brain injury are important for understanding frontal-dominant neurocognitive dysfunction in adult MMD. Pyramidal neocortical neurons (layers 3, 5, and 6) in the prefrontal cortex are known to be highly vulnerable to hypoxic-ischemic insults. $^{31,32}$ These hypoxic-ischemic insults can damage the brain and potentially induce neuronal death or regression of dendritic structure. ${ }^{8}$ We speculate that microstructural alteration precedes gross volumetric reductions in gray matter. Previous study has revealed gray matter atrophy in the posterior cingulate cortex, suggesting that more widespread gray matter changes might be observed in diffusion parameters, particularly in the frontal lobe. ${ }^{3}$ DKI could reveal early microstructural changes less constrained by partial volume effects. ${ }^{33}$ Nevertheless, despite discrete white matter damage, no substantial changes in diffusion parameters were found in the cortical gray matter in MMD. Pathologic tissue changes such as glial proliferation may underlie the lack of significant changes in the diffusion parameters of the cortices. A new diffusion MR imaging technique, including neurite orientation dispersion and density imaging, may detect subtle microstructural changes in the cortex and is potentially sensitive to initial ischemic changes before the overt volumetric reductions. ${ }^{34,35}$

The present study revealed microstructural change in the thalamus, a finding that has never been emphasized with regard to cognitive function in MMD. The mediodorsal thalamus connects to the prefrontal cortex. This is potentially important because innervation of the thalamoprefrontal circuit could modulate prefrontal neural circuits, which are associated with cognitive as well as affective performance. ${ }^{36}$

There are several limitations to this study. The statistical power to detect group differences in DKI parameters is significantly influenced by the number of subjects in a study. ${ }^{37}$ Therefore, the spatial characteristics of the DKI/DTI alterations found in this study may not represent the topography of ischemic burden in adult MMD. ${ }^{37}$ We extracted DKI/DTI values from the contrast $T$ maps generated from group comparisons between controls and patients to explore the relationship between abnormal diffusion parameters in patients and neuropsychological test performance. Although we found an association with neuropsychological test scores, voxel-based correlation analysis would per- 
A WAIS-III

ROI: Axial kurtosis
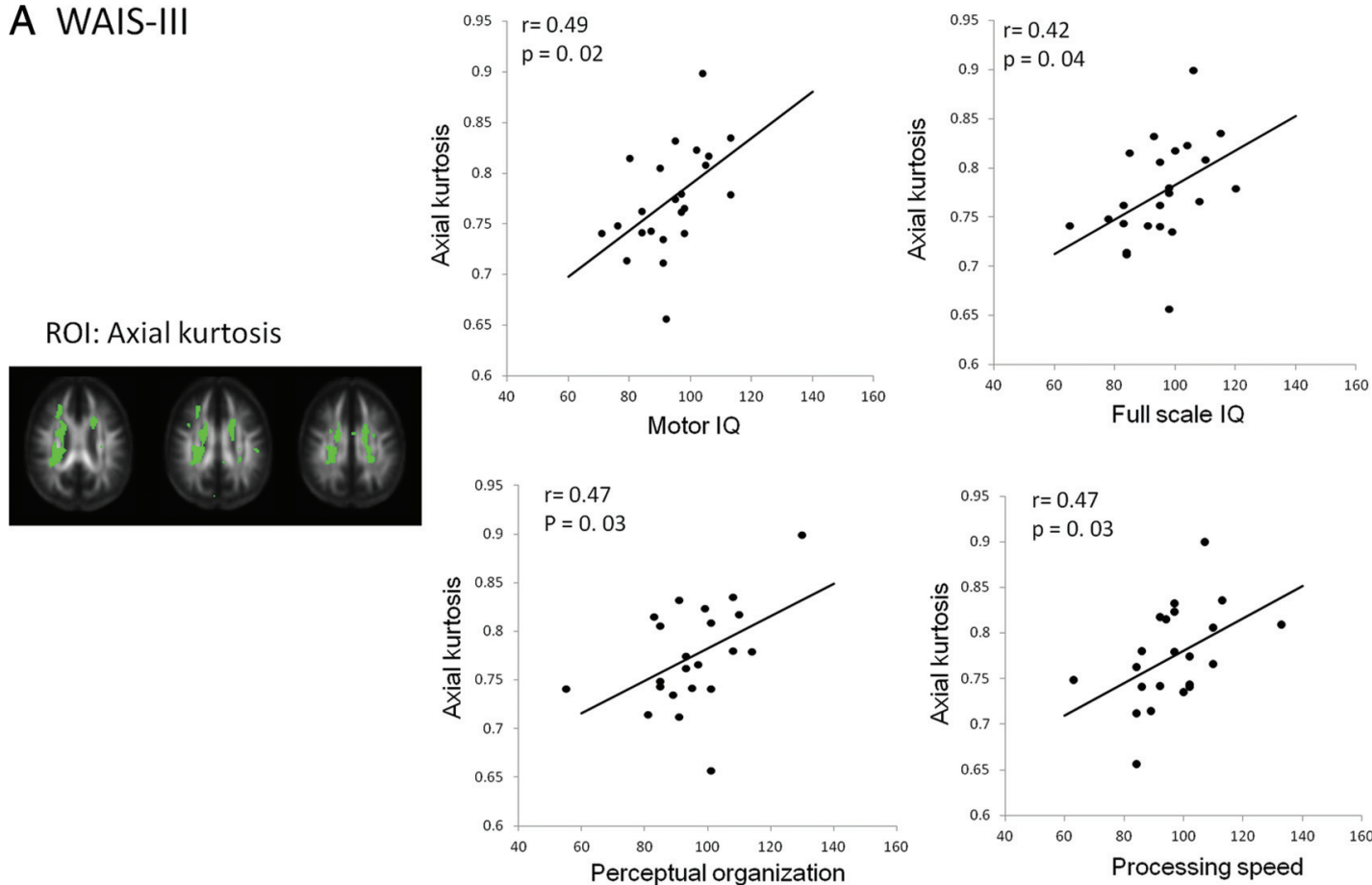

\section{B Trail making test $(B)$}

ROI: Mean kurtosis

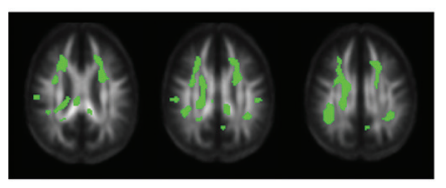

ROI: Fractional anisotropy

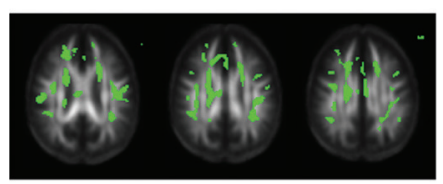

ROI: Mean diffusivity

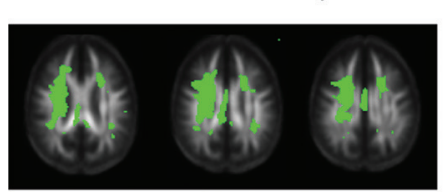

ROI: Radial diffusivity

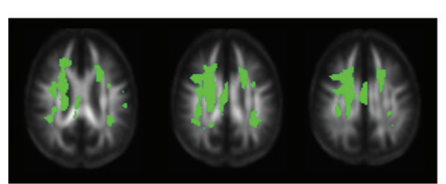

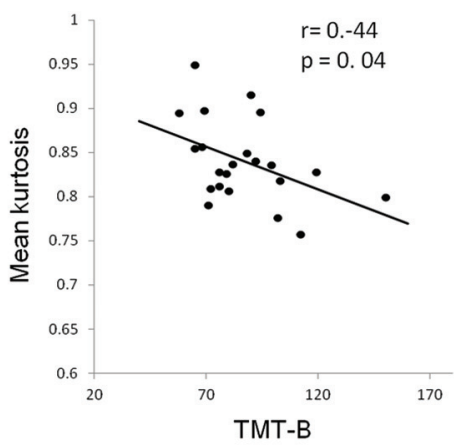
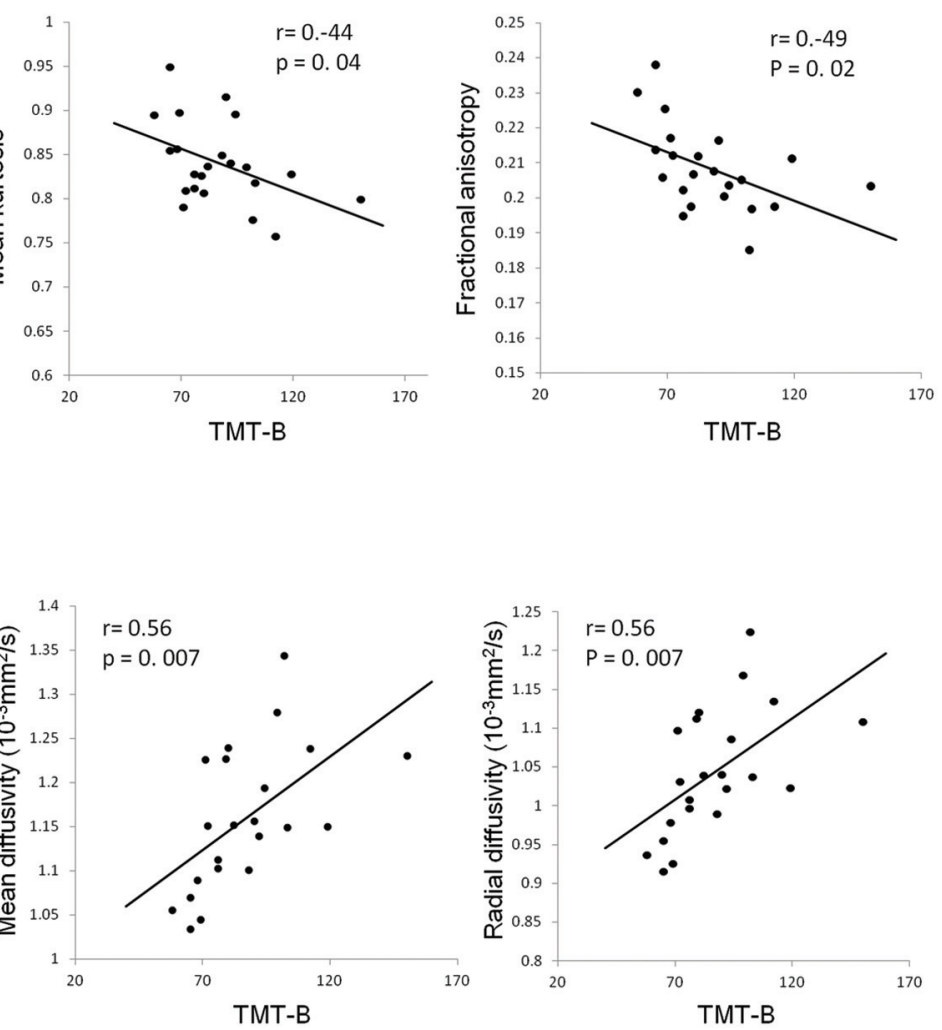

FIG 3. Scatterplots indicating a significant correlation between neuropsychological test performance and diffusion parameters. Pearson moment-production correlation coefficient $r$ and $P$ values are demonstrated in each scatterplot. ROls (green) for each parameter (AK, MK, FA, $\mathrm{MD}$, and RD) are demonstrated with FA template images generated from 23 controls and 23 patients. A, Performance scores evaluated on the Wechsler Adult Intelligent Scale-III are significantly associated with axial kurtosis. Axial kurtosis is positively correlated with full-scale IQ $(r=$ $0.42, P=.04)$ and subscores such as motor IQ $(r=0.49, P=.02)$, perceptual organization $(r=0.47, P=.03)$, and processing speed $(r=0.47, P=$ .03). $B$, Trail-Making Test, part B is inversely correlated with DKI/DTI parameters (MK; $r=-0.44, P=.04$; and FA; $r=-0.49, P=.02$ ) and positively correlated with DTI parameters (MD; $r=0.56, P=.007$; and RD; $r=0.56, P=.007$ ). 
Table 3: Correlations of diffusion parameters with neuropsychological examinations ${ }^{a}$

\begin{tabular}{lcccc}
\hline & WCST & Stroop & CPT & RST \\
\hline MK & - & $.004^{\mathrm{b}}$ & - & $.048^{\mathrm{b}}$ \\
RK & - & $.007^{\mathrm{b}}$ & - & - \\
AK & - & $.045^{\mathrm{c}}$ & - & $.036^{\mathrm{c}}$ \\
FA & - & $.024^{\mathrm{b}}$ & - & - \\
MD & - & $.012^{\mathrm{b}}$ & - & - \\
RD & - & $.014^{\mathrm{b}}$ & - & - \\
AD & - & $.031^{\mathrm{c}}$ & - & - \\
\hline
\end{tabular}

Note:-WCST indicates Wisconsin Card Sorting Test, Stroop; Stroop test.

${ }^{a}$ Data are $P$ values. Correlations of diffusional kurtosis imaging and diffusion tensor imaging parameters with neuropsychological examinations were evaluated in patients with MMD. Three diffusional kurtosis parameters (MK, RK, and AK) and 4 diffusion tensor parameters (FA, MD, RD, and AD) were analyzed. Diffusion parameters and locations that demonstrated significant relationships with clinical variables follow.

${ }^{\mathrm{b}}$ Two-tailed $t$ test.

cOne-tailed $t$ test.

mit an objective evaluation of the neural substrates associated with cognitive impairment. Finally, we used a combination of b-values of 0 and $1000 \mathrm{~s} / \mathrm{mm}^{2}$ for DTI. DTI parameters are reported as dependent on b-values. For DKI, the b-values of 0, 1000, and $2000 \mathrm{~s} / \mathrm{mm}^{2}$ used in the present study would be feasible for practical clinical applications. Nevertheless, a different combination of b-values and methods for parameter estimation could alter the relationship between DKI and DTI with regard to sensitivity and specificity, which requires further investigation. ${ }^{21}$

\section{CONCLUSIONS}

The results of the present study suggest an additional value of DKI as an adjunct to DTI. DKI parameters can become useful neuroimaging markers to track ischemic burden in adult MMD.

Disclosures: Ken Kazumata—RELATED: Grant: This study was supported by a grant from the Research Committee on Moyamoya Disease, sponsored by the Ministry of Health, Labor, and Welfare of Japan. * Khin K. Tha—RELATED: Grant: This work was supported (in part) by the Creation of Innovation Centers for Advanced Interdisciplinary Research Areas Program, Ministry of Education, Culture, Sports, Science, and Technology, Japan.* Masaki Ito—RELATED: Grant: Research Committee on Moyamoya Disease, sponsored by the Ministry of Health, Labor, and Welfare of Japan*; UNRELATED: Grants/Grants Pending: Masaki Ito was funded by a fellowship (grant) $¥ 2,000,000$ J (approximately US $\$ 18,000$ ) for 1 year from SENSHIN Medical Research Foundation donated by the Mitsubishi Corporation, Japan. Funding began April 1, 2015. Takeo Abumiya—RELATED: Grant: grant from the Research Committee on Moyamoya Disease, sponsored by the Ministry of Health, Labor, and Welfare of Japan. * Money paid to the institution.

\section{REFERENCES}

1. Kuroda S, Houkin K. Moyamoya disease: current concepts and future perspectives. Lancet Neurol 2008;7:1056-66 CrossRef Medline

2. Karzmark P, Zeifert PD, Bell-Stephens TE, et al. Neurocognitive impairment in adults with Moyamoya disease without stroke. Neurosurgery 2012;70:634-38 CrossRef Medline

3. Kazumata K, Tha KK, Narita H, et al. Chronic ischemia alters brain microstructural integrity and cognitive performance in adult Moyamoya disease. Stroke 2015;46:354-60 CrossRef Medline

4. Tha KK, Terae S, Nakagawa S, et al. Impaired integrity of the brain parenchyma in non-geriatric patients with major depressive disorder revealed by diffusion tensor imaging. Psychiatry Res 2013;212: 208-15 CrossRef Medline

5. Basser PJ, Mattiello J, LeBihan D. MR diffusion tensor spectroscopy and imaging. Biophys J 1994;66:259-67 Medline

6. Jensen JH, Helpern JA, Ramani A, et al. Diffusional kurtosis imaging: the quantification of non-gaussian water diffusion by means of magnetic resonance imaging. Magnetic Reson Med 2005; 53:1432-40 Medline
7. Back SA. Cerebral white and gray matter injury in newborns: new insights into pathophysiology and management. Clin Perinatal 2014;41:1-24 CrossRef Medline

8. Zhang S, Boyd J, Delaney K, et al. Rapid reversible changes in dendritic spine structure in vivo gated by the degree of ischemia. $\mathrm{JNeu}$ rosci 2005;25:5333-38 Medline

9. Fieremans E, Jensen JH, Helpern JA. White matter characterization with diffusional kurtosis imaging. Neuroimage 2011;58:177-88 CrossRef Medline

10. Li X, Gao J, Hou X, et al. Diffusion kurtosis imaging with tractbased spatial statistics reveals white matter alterations in preschool children. Conf Proc IEEE Eng Med Biol Soc 2012;2012:2298-301 CrossRef Medline

11. Coutu JP, Chen JJ, Rosas HD, et al. Non-Gaussian water diffusion in aging white matter. Neurobiol Aging 2014;35:1412-21 CrossRef Medline

12. Gong NJ, Wong CS, Chan CC, et al. Aging in deep gray matter and white matter revealed by diffusional kurtosis imaging. Neurobiol Aging 2014;35:2203-16 CrossRef Medline

13. Caverzasi E, Henry RG, Vitali P, et al. Application of quantitative DTI metrics in sporadic CJD. Neuroimage Clin 2014;4:426-35 CrossRef Medline

14. Weber RA, Hui ES, Jensen JH, et al. Diffusional kurtosis and diffusion tensor imaging reveal different time-sensitive strokeinduced microstructural changes. Stroke 2015;46:545-50 CrossRef Medline

15. Grinberg F, Farrher E, Ciobanu L, et al. Non-Gaussian diffusion imaging for enhanced contrast of brain tissue affected by ischemic stroke. PLoS One 2014;9:e89225 CrossRef Medline

16. Umesh Rudrapatna S, Wieloch T, Beirup K, et al. Can diffusion kurtosis imaging improve the sensitivity and specificity of detecting microstructural alterations in brain tissue chronically after experimental stroke? Comparisons with diffusion tensor imaging and histology. Neuroimage 2014;97:363-73 CrossRef Medline

17. Tabesh A, Jensen JH, Ardekani BA, et al. Estimation of tensors and tensor-derived measures in diffusional kurtosis imaging. Magn Reson Med 2011;65:823-36 CrossRef Medline

18. Reijmer YD, Leemans A, Heringa SM, et al; Vascular Cognitive Impairment Study group. Improved sensitivity to cerebral white matter abnormalities in Alzheimer's disease with spherical deconvolution based tractography. PLoS One 2012;7:e44074 CrossRef Medline

19. Wakana S, Jiang H, Nagae-Poetscher LM, et al. Fiber tract-based atlas of human white matter anatomy. Radiology 2004;230:77-87 Medline

20. Cheng HL, Lin CJ, Soong BW, et al. Impairments in cognitive function and brain connectivity in severe asymptomatic carotid stenosis. Stroke 2012;43:2567-73 Medline

21. Schmidt R, Seiler S, Loitfelder M. Longitudinal change of small-vessel disease-related brain abnormalities. J Cereb Blood Flow Metab 2015 Apr 22. [Epub ahead of print] CrossRef Medline

22. Kamagata K, Tomiyama H, Hatano T, et al. A preliminary diffusional kurtosis imaging study of Parkinson disease: comparison with conventional diffusion tensor imaging. Neuroradiology 2014; 56:251-58 CrossRef Medline

23. Fieremans E, Benitez A, Jensen JH, et al. Novel white matter tract integrity metrics sensitive to Alzheimer disease progression. AJNR Am J Neuroradiol 2013;34:2105-12 CrossRef Medline

24. Steven AJ, Zhuo J, Melhem ER. Diffusion kurtosis imaging: an emerging technique for evaluating the microstructural environment of the brain. AJR Am J Roentgenol 2014;202:W26-33 CrossRef Medline

25. Cechetti F, Pagnussat AS, Worm PV, et al. Chronic brain hypoperfusion causes early glial activation and neuronal death, and subsequent long-term memory impairment. Brain Res Bull 2012;87: 109-16 CrossRef Medline 
26. Jbabdi S, Behrens TE, Smith SM. Crossing fibres in tract-based spatial statistics. Neuroimage 2010;49:249-56 CrossRef Medline

27. Gläscher J, Rudrauf D, Colom R, et al. Distributed neural system for general intelligence revealed by lesion mapping. Proc Natl Acad Sci U S A 2010;107:4705-09 CrossRef Medline

28. Deary IJ, Weiss A, Batty GD. Intelligence and personality as predictors of illness and death: how researchers in differential psychology and chronic disease epidemiology are collaborating to understand and address health inequalities. Psychol Sci Public Interest 2010;11: 53-79 CrossRef Medline

29. Barbey AK, Colom R, Solomon J, et al. An integrative architecture for general intelligence and executive function revealed by lesion mapping. Brain 2012;135:1154-64 CrossRef Medline

30. Kurumatani T, Kudo T, Ikura Y, et al. White matter changes in the gerbil brain under chronic cerebral hypoperfusion. Stroke 1998;29: 1058-62 Medline

31. Lin CS, Polsky K, Nadler JV, et al. Selective neocortical and thalamic cell death in the gerbil after transient ischemia. Neuroscience 1990; 35:289-99 Medline

32. Fukuda A, Muramatsu K, Okabe A, et al. NMDA receptor-mediated differential laminar susceptibility to the intracellular $\mathrm{Ca} 2+$ accu- mulation induced by oxygen-glucose deprivation in rat neocortical slices. J Neurophysiol 1998;79:430-38 Medline

33. Yang AW, Jensen JH, Hu CC, et al. Effect of cerebral spinal fluid suppression for diffusional kurtosis imaging. J Magn Reson Imaging 2013;37:365-71 CrossRef Medline

34. Jelescu IO, Veraart J, Adisetiyo V, et al. One diffusion acquisition and different white matter models: how does microstructure change in human early development based on WMTI and NODDI? Neuroimage 2015;107:242-56 CrossRef Medline

35. Zhang H, Schneider T, Wheeler-Kingshott CA, et al. NODDI: practical in vivo neurite orientation dispersion and density imaging of the human brain. Neuroimage 2012;61:1000-16 CrossRef Medline

36. Ferguson BR, Gao WJ. Development of thalamocortical connections between the mediodorsal thalamus and the prefrontal cortex and its implication in cognition. Front Hum Neurosci 2014;8:1027 Medline

37. Szczepankiewicz F, Lätt J, Wirestam R, et al. Variability in diffusion kurtosis imaging: impact on study design, statistical power and interpretation. Neuroimage 2013;76:145-54 CrossRef Medline 